\title{
Body Wall Force Sensor for Simulated Minimally Invasive Surgery : Application to Fetal Surgery
}

\author{
Allan Javaux ${ }^{1}$, Laure Esteveny ${ }^{1}$, David Bouget ${ }^{1}$, Caspar Gruijthuijsen ${ }^{1}$, Danail Stoyanov ${ }^{2}$, Tom Vercauteren ${ }^{3}$, \\ Sebastien Ourselin ${ }^{3}$, Dominiek Reynaerts ${ }^{1}$, Kathleen Denis ${ }^{1}$, Jan Deprest ${ }^{3,4}$ and Emmanuel Vander Poorten ${ }^{1}$
}

\begin{abstract}
Surgical interventions are increasingly executed minimal invasively. Surgeons insert instruments through tiny incisions in the body and pivot slender instruments to treat organs or tissue below the surface. While a blessing for patients, surgeons need to pay extra attention to overcome the fulcrum effect, reduced haptic feedback and deal with lost hand-eye coordination. The mental load might make it difficult to pay sufficient attention to the forces that are exerted on the body wall. In delicate procedures such as fetal surgery, this might be problematic as irreparable damage could cause premature delivery. As a first attempt to quantify the interaction forces applied on the patient's body wall, a novel 6 degrees of freedom force sensor was developed for an ex-vivo set up. The performance of the sensor was characterised. User experiments were conducted by 3 clinicians on a set up simulating a fetal surgical intervention. During these simulated interventions, the interaction forces were recorded and analysed when a normal instrument was employed. These results were compared with a session where a flexible instrument under haptic guidance was used. The conducted experiments resulted in interesting insights in the interaction forces and stresses that develop during such difficult surgical intervention. The results also implicated that haptic guidance schemes and the use of flexible instruments rather than rigid ones could have a significant impact on the stresses that occur at the body wall.
\end{abstract}

\section{INTRODUCTION}

Over the past decades, technological developments greatly impacted the quality of medicine [1]. A typical example is minimally invasive surgery (MIS) where thanks to the availability of high quality endoscopes, it became possible to conduct interventions without needing large incisions. MIS greatly improved patient outcome, causing smaller scars and offering shorter recovery [2]. However, MIS did complicate the surgeon's life, requiring to overcome the loss of haptic or tactile feedback or to deal with issues such as a lost handeye coordination, a fulcrum effect and a varying leverage arm [3], [4].

Twin-twin transfusion syndrome (TTTS) [5] is a particularly difficult minimally invasive laser procedure in fetal surgery, requiring extremely precise motion in a very fragile environment. Currently fetoscopy is done manually, but surgeons experience significant problems to reach certain areas

Research funded by Wellcome Trust and EPSRC, project GIFT-Surg

${ }^{1}$ KU Leuven, Department of Mechanical Engineering, Leuven, Belgium allan.javaux@kuleuven.be

${ }^{2}$ University of College London, Department of Computer Science, London, United Kingdom

${ }^{3}$ University of College London, Department of Medical Physics \& Biomedical Engineering, University College London, London, United Kingdom

${ }^{4}$ University Hospital Leuven, Leuven, Belgium

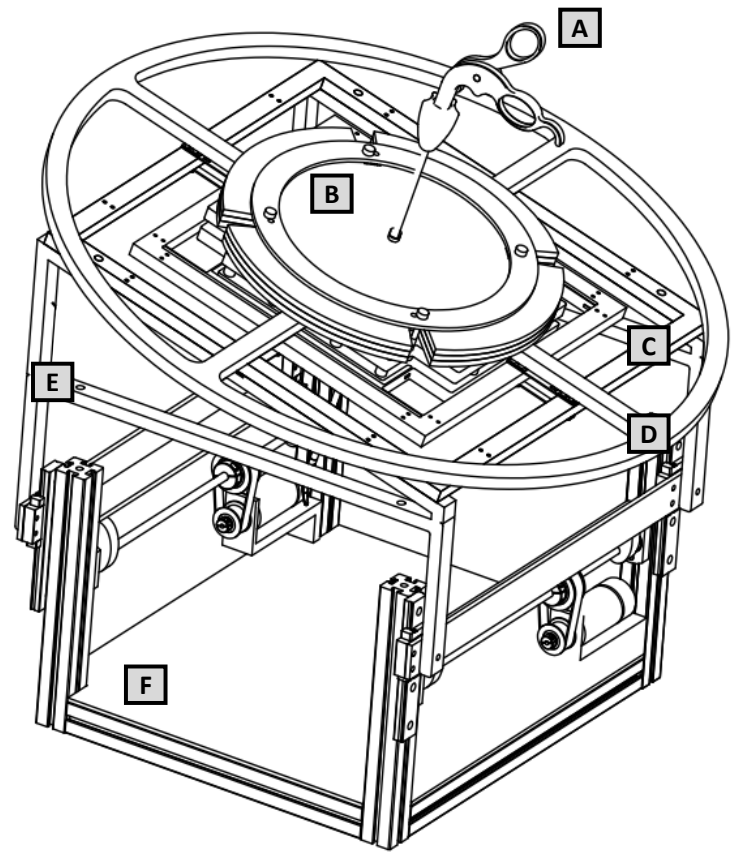

Fig. 1: Envisioned ex-vivo set up with force sensor : a) surgical instrument, b) body wall, c) force sensor, d) supporting frame for tissue-sheet e) supporting frame for force sensor f) adaptable platform allowing the body wall to move and follow eventually physiological motion.

of the placenta without applying too large stress upon the tissue and/or instrument. Depending on the surgeon's motion, large stresses can develop e.g. at the incision. Such stresses might induce iatrogenic premature preterm rupture of the membrane (iPPROM) which might cause premature birth, hence reducing drastically the survival rate of the twins [6].

To investigate the first hypothesis and in general to obtain a better understanding of the levels of stress that are being applied on the body wall, i.e. the several layers of tissue separating the patient's internals from the outside world, a dedicated force sensor has been designed for an ex-vivo set up. Fig.1 illustrates how the set up is envisioned. A piece of synthetic or real body wall is attached to the force sensor such that an instrument can be inserted via a cannula through the tissue. All forces that are applied upon the instrument now travel through the tissue to the force/torque sensor and then to the ground. Rather than directly measuring in-vivo, the sensor is designed for ex-vivo measurements as this 
poses less practical, ethical, regulatory or safety concerns. The sensor is considered helpful to determine approximate force/torque levels during simulated interventions and is also going to be useful when investigating the benefit of robotic assistance or of dedicated flexible instruments. However, in order to draw correct conclusions from an ex-vivo set up, efforts must be done to keep the system as close as possible to reality.

In MIS, surgical tools are inserted via a cannula which on its turn is inserted in a small incision in the patient body wall. As the instrument is inserted or retracted, friction develops between the instrument and the cannula. This force masks the actual interaction forces that are generated in contact with the targeted tissue. Researchers have devised different force/torque sensors to measure directly inside the body i.e. beyond the incision point. As the sensor has to pass through the cannula at some point, the former should thus be very compact and mounted closeby the instrument tip. Small force sensors were made by a.o. Peirs, Puangmali and Seibold et al., [7], [8], [9]. Most are based on compliant mechanisms [10] that deform when forces are applied. In [7], such mechanism is used with optical fibers. A flexible Stewart Platform coupled with strain gauges has been reported by Seibold et al. [9]. Puangmali et al. developed an optical fiber based sensor to detect axial forces applied at the tip in [8]. Apart from practical issues to get these sensors robust and sterilisable, these do not allow the estimation of the stress on the body wall which is of interest in TTTS.

Sensors that measure forces and torques at the incision point of the cannula have been developed in the past [11], [12], [13]. Commercial 6 Degree Of Freedom (DOFs) sensors have been directly integrated at the cannula[12] while a combination of force sensors (one at the cannula and one at the instrument holder) was used to reconstruct 3 DOFs force components at the tool tip by Shimachi et al. [11] or along the direction of motion by Willaert et al. [13]. The objective of these works was to reconstruct the force/torque at the tool tip. The forces and torques at the incision point were seen as disturbances that were measured so as to cancel out their effect. In this work, these forces/torques form exactly the object of interest as they could be the cause of iPPROM after TTTS. The methods by Shimachi, Zemiti and Willaert et al. can not be used for this study as the sensor itself should not have any affect on the interaction with the tissue.

Our contributions in this study are the following:

- presentation and experimental validation of an innovative 6 DOFs body wall force sensor for an ex-vivo set up

- integration of the sensor in a simulated set up

In section II, an in-depth description of the proposed body wall force sensor is presented. Section III introduces the followed calibration procedure and includes the characterization of the sensor. Section IV shows some experimental results where the sensor is used in a simulated TTTS intervention.
Finally, perspectives of future work can be found in Section $\mathrm{V}$.

\section{THE BODY WALL FORCE SENSOR}

\section{A. Design requirements}

A novel sensor is to be designed such that it can measure the forces and torques applied on the body wall during fetal MIS. The force sensor should allow unhindered cannula and/or instrument motion (inclination $\pm 80^{\circ}$ ) around the incision point comparable to motion during a real TTTS intervention. In order to capture all intentional motion/forces, the bandwidth should be $10 \mathrm{~Hz}$ or higher [14]. As to the best of our knowledge, no measurements of forces and torques during TTTS have been done before. A small test was conducted to get an estimate of a reasonable force sensor range. For this, we used the force needed to insert a fetoscope inside a cannula as a measure. In TTTS, fetoscopes are typically tightly fit in the cannula to avoid leakage of the amniotic fluid. A result is that, especially during insertion and retraction of the instrument, large (friction) forces arise. A dynamometer measured a force of approximately $10 \mathrm{~N}$ when exerting the scope. In addition, the force sensor should be able to support a body wall weighing up to $2 \mathrm{~kg}$. For safety reasons and accounting for the variable inclination of the tool, the following force ranges were considered: 0-40 N along the vertical axis $\vec{z}$ and $0-20 \mathrm{~N}$ along the two other orthogonal directions, $\vec{x}$ and $\vec{y}$. It was also considered that the incision point should be able to move a centimeter or so from its initial position, due to the tissue elasticity. Thus, a potential deviation of $10 \mathrm{~mm}$ from the incision point was considered, leading to the following torque ranges: $0-0.2 \mathrm{Nm}$ around $\vec{z}$ and $0-0.4 \mathrm{Nm}$ around $\vec{x}$ and $\vec{y}$. An accuracy of $1 \%$, comparable to standard force sensors is considered sufficient. The main specifications are summarized in Table I.

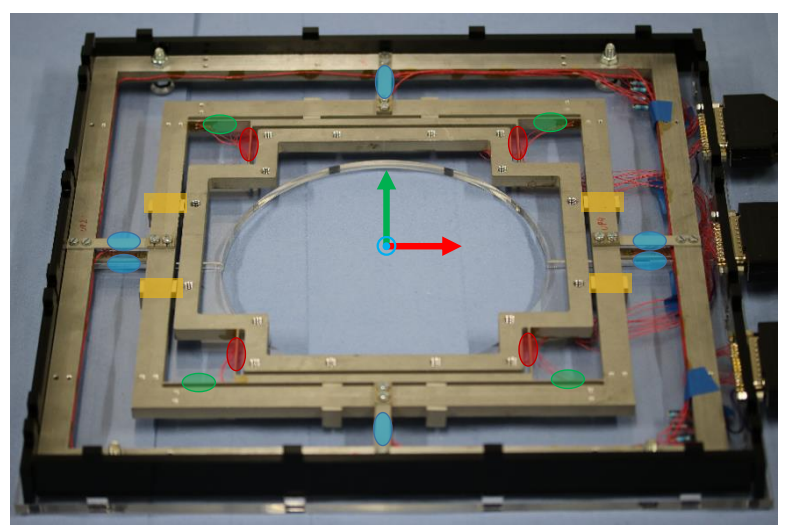

Fig. 2: 6 DOFs force sensor composed of three parts : inner, middle, and outer. Red, green and blue colors indicate the flexible beams, on which 16 strain gauges are positioned (Two blue beams aren't visible with this point of view). Safety stops are highlighted in yellow. A global frame is represented with axis $\mathrm{x}$ (red), $\mathrm{y}$ (green) and $\mathrm{z}$ (blue). 
TABLE I: Main sensor specifications.

\begin{tabular}{ll}
\multicolumn{2}{c}{ Specifications } \\
\hline DOFs & 6 \\
Bandwidth & {$[0-10] \mathrm{Hz}$} \\
Vertical force $\vec{z}$ & {$[0-40] \mathrm{N}$} \\
Planar forces $\vec{x}, \vec{y}$ & {$[0-20] \mathrm{N}$} \\
Torque along vertical axis $\vec{z}$ & {$[0-0.2] \mathrm{Nm}$} \\
Torque in plain $\vec{x}, \vec{y}$ & {$[0-0.4] \mathrm{Nm}$} \\
\hline
\end{tabular}

\section{B. Mechanical Design}

1) Working principle: Fig. 2 shows the proposed sensor. It is a compliant mechanism composed of an outer, middle and inner frame. The inner ring contains a large opening. A clamping ring can be used to fix the tissue and clamp it with a certain appropriate tension to the inner frame, as done in Section IV. An incision and a cannula are preferably made/inserted in the center of this tissue. The inner frame's dimensions determine thus the workspace (inclination) of the instrument. This explains the large size of the inner ring. The outer and middle frames are rigid and connected to each other by means of flexible beams (highlighted in red, green and blue). The outer frame represents the base while the inner frame is attached to the body wall. Thus, all forces that are applied upon the instrument now travel through the tissue to the force/torque sensor and then to the ground. The flexible beams have been positioned such that the force/torque components could theoretically be determined independently. Indeed, forces acting along $\vec{x}, \vec{y}$ and $\vec{z}$ would only put a mechanical strain on the beams in, respectively, red, green and blue (Fig.2). By measuring these strains and combining them, it becomes possible to determine the forces and torques applied on the inner frame.

2) Prototype: The sensor is made out of Aluminium 7075 which is chosen for its high ratio of yield strength to Young's modulus ensuring a large linear range of deformation [10]. Furthermore, this material improves repeatability and limits non-linear effects since creep and stress relaxation would be almost non-existing. To ensure a sufficiently large inner area, the size of the inner plate follow the dimensions of the outer frame $350 \times 350 \times 10 \mathrm{~mm}$. The beams have been designed such that when under maximal load, the stresses on the structure remain below the material's admissible stress. The inner and middle frames were made in a monolithic structure to suppress any backlashes and by electrical discharge machining (EDM) to minimize possible stresses induced by the manufacturing process. The flexible beams connecting the middle and outer frame are tightly squeezed between intermediate plates by four nuts and bolts per beam to ensure a behavior similar to the one of a monolithic structure (linearity, backlash-free). Safety stops (yellow in Fig. 2) were also added to protect the sensor against possible overload.

3) Force and torque estimation: Due to their compactness and precision, strain gauges are positioned along each flexible beam to assess these strains. Under a given deflection of a beam, the resistance of the associated strain gauge changes, leading to a voltage output that monotonically rises with deformation. Given the design, the vector $\tau$ of forces and torques applied on the inner frame is linked to a combination of strain gauge output voltages $V$ by the following relationship:

$$
\tau=\mathbf{K} V
$$

with the wrench $\tau=\left[F_{x}, F_{y}, F_{z}, M_{x}, M_{y}, M_{z}\right]^{T}$ where $F_{x}, F_{y}$ and $F_{z}$ represent the forces applied on the sensor along respectively $\vec{x}, \vec{y}$ and $\vec{z} . M_{x}, M_{y}$ and $M_{z}$ represent the torques around respectively axis $\vec{x}, \vec{y}$ and $\vec{z}$. $\mathbf{K}$ is a $6 \times 6$ matrix that should theoretically be diagonal. However, manufacturing precision and non-exact positioning of the gauges inevitably lead to possible coupling. The strain gauges are wired to 16 National Instrument Wheatstone quarter-bridges. The data is captured in real-time with Labview at $100 \mathrm{~Hz}$, providing sufficient bandwidth for adding a noise filter and still respecting the desired specifications.

\section{Calibration \& Characterization}

A dedicated test-bench has been developed to calibrate and then characterize the sensor. After presenting the test-bench, the calibration is described. Then, the focus is shifted to the performance that could be reached.

\section{A. Test-bench presentation}

Fig. 3 shows the developed calibration test-bench. A 1 DOF load cell (BCM Single Point Load Cell Model 1668(S) - $1 \mathrm{~kg}$ ) is attached to a linear stage, actuated by means of a ball screw and rotary motor (Maxon Motor RE 30 - 268193). This set up allows to thoroughly control the force applied on the force sensor of the developed sensor throughout a custom-made force transmitter firmly attached to the inner frame. Each component of the force/torque can then be

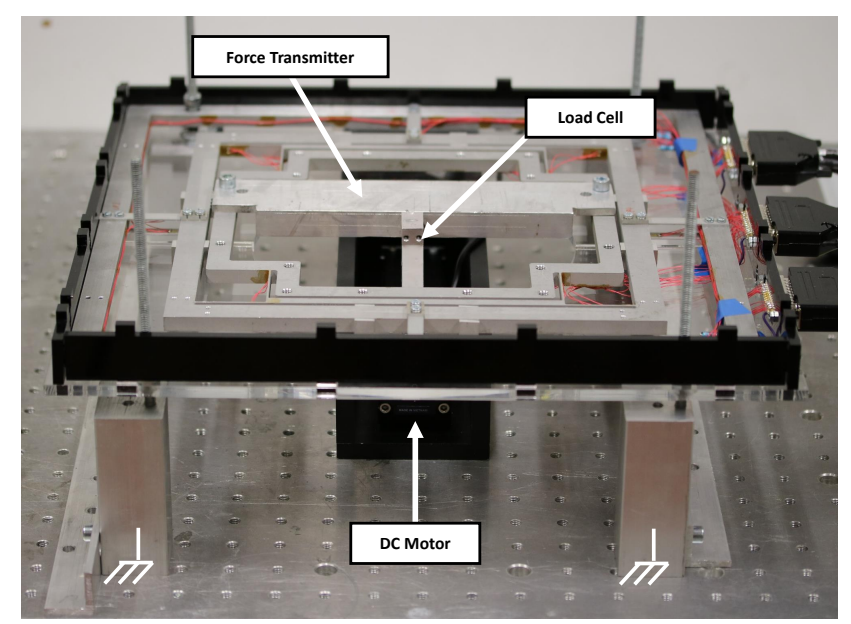

Fig. 3: Test-bench in a configuration for calibrating and characterizing the component $F_{y}$ of the sensor. The DC motor deploys the load cell such that a particular force is applied on the force transmitter which is firmly attached to the inner part of the force sensor. 
independently obtained by adjusting the application point and direction of the force on the force transmitter. Indeed, regarding moments, a force is applied at a certain distance (lever) from the sensor (i.e.. no pure moments are applied). Fig. 3 shows the configuration that was used to apply forces in the $(\vec{x}, \vec{y})$ plane. The test-bench was put vertical to obtain the other components.

The measurement software was implemented in Labview. A conventional current controller was first implemented, but friction in the transmission prevented to set forces adequately. Therefore the controller was replaced by a force controller that used the force measurements of the $1 \mathrm{DOF}$ load cell in a closed feedback loop to precisely set the force applied on the sensor.

\section{B. Calibration}

To calibrate the sensor, the test bench was set up in 6 different configurations applying either pure forces along each axis or applying a force at a certain offset (which corresponds to a combined force and torque loading). For each of these configurations, a ramp in force varying from [0-10] $\mathrm{N}$ for the force components and from [0-5] $\mathrm{N}$ with an offset of $20 \mathrm{~mm}$ for the torque components. Knowing the application point of the force, the matrix $\tau_{m}$ with dimension $n \times 6$ is then obtained from the $n$ load cell measurements. The voltages over all 16 strain gauges are measured and combined to form a reduced matrix $\mathbf{V}_{m}$ with dimension $n \times 6$. In total, 7500 measurements were taken into account to calculate the calibration matrix $\mathbf{K}_{m}$ :

$$
\mathbf{K}_{m}=\left(\mathbf{V}_{m}^{+} \cdot \tau_{m}\right)^{T}
$$

with $\mathbf{V}_{m}^{+}$the Moore-Penrose pseudo-inverse of the measurements matrix $\mathbf{V}_{m}$, which corresponds to using the least squares method to fit the strains in each configuration.

\section{Characterization}

After calibration, the same test-bench was used to characterize the sensor's performance. The performance was evaluated independently per component $F_{x}, F_{y}, F_{z}, M_{x}, M_{y}$ and $M_{z}$. Following paragraphs describe the results which are also summarized in Tables II and III.

1) Accuracy and precision: The sensor's accuracy and precision was evaluated by, again, applying a force ramp from $0.5 \mathrm{~N}$ to $10 \mathrm{~N}$ (changing at a rate of $0.5 \mathrm{~N} / \mathrm{s}$ ) for each component, and using the $20 \mathrm{~mm}$ lever when exciting torques. Such cycle was repeated 50 times per component. The root mean square (RMS) of the difference between the load-cell and the sensor measurements was used as a measure of the sensor's accuracy. The standard deviation was then computed for each force target over all cycles. To characterize the sensor's precision, the maximum encountered standard deviation was employed.

The results are summarized in Table II. By considering separately the range for each axis of the force sensor, an accuracy and precision of $0.21 \% \pm 0.23 \%$ with the $F_{y}$ component was obtained, while for $F_{x}$, the accuracy was $2.06 \% \pm 1.63 \%$. Since the design is practically symmetric
TABLE II: Result of Performance Analysis Part I - Accuracy, Precision, Noise, Drift

\begin{tabular}{ccccc}
\hline & $\begin{array}{c}\text { RMS Error } \\
{[\mathrm{N}-\mathrm{Nm}]}\end{array}$ & $\begin{array}{c}\text { Max } \sigma \\
{[\mathrm{N}-\mathrm{Nm}]}\end{array}$ & $\begin{array}{c}\text { SNR } \\
{[-]}\end{array}$ & $\begin{array}{c}\text { Drift } \\
{[\mathrm{N}-\mathrm{Nm}]}\end{array}$ \\
\hline$F_{x}$ & 0.412 & 0.325 & 136.84 & 0.057 \\
$F_{y}$ & 0.042 & 0.047 & 224.56 & 0.020 \\
$F_{z}$ & 0.079 & 0.094 & 93.75 & 0.002 \\
$M_{x}$ & 0.007 & 0.009 & 8.41 & 0.003 \\
$M_{y}$ & 0.027 & 0.171 & 6.33 & 0.015 \\
$M_{z}$ & 0.021 & 0.009 & 11.09 & 0.001 \\
\hline
\end{tabular}

TABLE III: Result of Performance Analysis Part II - Coupling between components

\begin{tabular}{c|cccccc} 
& $F_{x}$ & $F_{y}$ & $F_{z}$ & $M_{x}$ & $M_{y}$ & $M_{z}$ \\
\hline$F_{x}$ Ref & - & 0.048 & 0.059 & 0.007 & 0.007 & 0.055 \\
$F_{y}$ Ref & 0.051 & - & 0.059 & 0.006 & 0.008 & 0.003 \\
$F_{z}$ Ref & 0.041 & 0.042 & - & 0.009 & 0.007 & 0.004 \\
$M_{x}$ Ref & 0.045 & 0.067 & 0.057 & - & 0.007 & 0.003 \\
$M_{y}$ Ref & 0.131 & 0.039 & 0.056 & 0.006 & - & 0.009 \\
$M_{z}$ Ref & 0.268 & 0.026 & 0.057 & 0.006 & 0.007 & -
\end{tabular}

for each direction, equal responses and accuracy would be expected. This was also confirmed through a finite element analysis. For this reason, it is believed that the difference in quality function of the direction may result from the manufacturing/assembly of the sensor and/or the mounting on the test rig for the calibration and performance analysis. This is open for further investigations but at this point, it was judged that the quality is sufficient to engage a more sophisticated human-in-the-loop experiment to highlight the potential of the force sensor and understand if the current obtained accuracy is actually enough.

2) Noise and drift: To quantify the noise and the sensor drift, a constant mid-range force of $5 \mathrm{~N}$ was continuously applied for 10 seconds and 10 minutes, respectively. The signal to noise ratio (SNR) was calculated. To describe the drift, the difference between initial and final error between the force measured by the load cell versus the one measured by the force sensor was used. Table II shows that forces are relatively less affected by noise compared to moments. Drift is mainly present in component $M_{y}$. For short experiments of less than 10 minutes, as realized for the user experiments hereafter, this will not be a problem.

3) Coupling: The coupling between the different forcetorque components that remained after calibration was investigated next. A force of $10 \mathrm{~N}$ was applied for 10 seconds with the appropriate lever. The RMS of the other components was calculated and reported in Table III. Overall, one can say that the resulting coupling remains small while only for the moments $M_{y}$ and $M_{z}$ there is some coupling with $F_{x}$. This may once again be explained by the misalignment of the set up or manufacturing issues. 


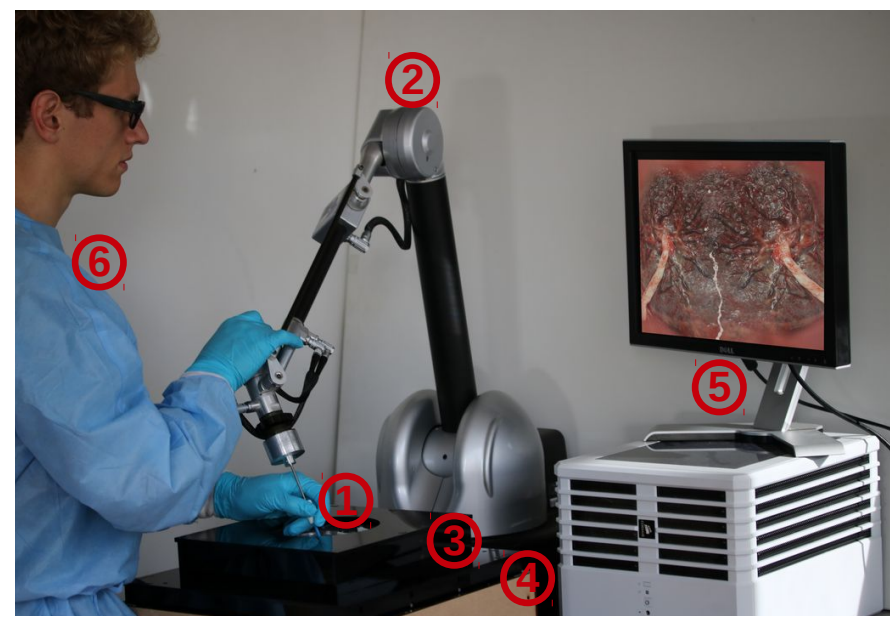

(a)

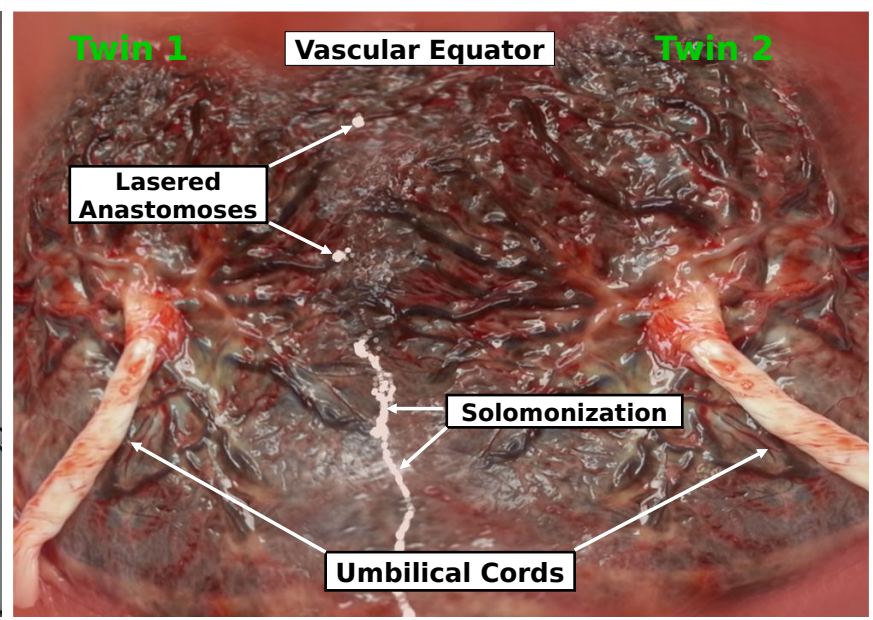

(b)

Fig. 4: Experimental set up for performing a virtual TTTS intervention. (a) Overall view of the experimental set up with 1) fetoscope replica, 2) Haption robotic arm, 3) body wall phantom covered with a protection case, 4) force sensor, 5) screen displaying a simulated surgical environment, 6) surgeon. (b) Zoom-in on the simulated surgical environment with the labelled anatomical structures.

\section{Simulation OF TWIN-TO-TWIN TRANSFUSION SYNDROME}

This section describes the outcome of a first validation study of the force sensor in a simulated environment. A set up simulating TTTS was prepared. Apart from an improved insight into the forces/torques applied during TTTS, we also hoped to learn whether the force information could potentially give us some additional insight in the displayed skills or whether there was any benefit in using a particular instrument or guidance scheme.

\section{A. Experimental set up}

The designed experimental set up, as shown in Fig. 4a, consists of a fetoscope, a robotic arm, a body wall phantom, a force sensor and a simulated environment. A foot pedal is provided as well with the set up (not shown in figure). These components are explained next.

1) Fetoscope: The employed instrument is composed of 2 parts: one physical and one virtual. A replica of a clinical fetoscope without its features, i.e. camera and laser, is handled by the operator. The physical fetoscope's pose is measured and a Virtual Reality (VR) system [15], depending on the motion of the fetoscope, adjusts the images displayed on the screen to simulate a fetoscope imerged in a real womb. Also, the virtual therapeutic laser is simulated. The virtual laser can be activated by pressing a foot pedal which will then cause an ablation appearing at the targeted spot on the screen. In this virtual setting, we experimented with different configurations of fetoscopes. A classic rigid fetoscope was prepared as well as a fetoscope with a virtual flexible tip. With the latter, it is possible to adjust unidirectionally the bending angle of the distal section in the virtual world. The simulated view from the scope is computed based on the state (pose and bending if present) of the fetoscope and displayed on an external monitor placed in front of the user.

2) Robotic arm: The surgical instrument is attached to the 6D Haption Virtuose ${ }^{1}$. Thanks to its combination of encoders and motors, the instrument pose can be retrieved and sent to the VR system. The instrument is co-manipulated by surgeon and the Virtuose. The latter can be programmed to provide variable levels and types of assistance (haptic guidance schemes) by inducing forces on the instrument. A guidance scheme can be combined with automatic control of the distal fetoscope DOFs in the case of the flexible fetoscope. For example, one of the implemented guidance schemes will perform automatic rotation of the instrument along its axis and actuate the virtual distal DOF to maintain the instrument's tip perpendicular to the placental plane. The Virtuose was also programmed to perform gravity compensation and recreate a damping effect on the instrument motion.

3) Body wall phantom: As explained during the introduction, even though the experiments are ex-vivo, the handling of the instrument must also be subjected to the similar haptic feeling present in-vivo. For that purpose, the body wall phantom must replicate the different layers of skin, fat, muscle, a uterus, and the fetal membranes. This is done by stacking several layers of synthetic material (EcoFlex 0050) together forming a total width of $40 \mathrm{~mm}$. A $3 \mathrm{~mm}$ diameter hole was made in the center to allow insertion of the instrument shaft. Clinicians indicated that they perceived this physical interaction to correspond fairly well to the feeling during real fetal interventions.

4) Force sensor: Our proposed force sensor as described in Section II was employed. The body wall phantom was clamped between the inner plate of the sensor and a Plexiglas

\footnotetext{
${ }^{1}$ Haption SA. Laval, France
} 
plate with screws holding everything together.

5) Simulated surgical environment: The main anatomical structures were simulated by the VR system [15] as visible in Fig. 4b. The simulation allows to position the placenta at different locations and can also vary the number and location of anastomoses to be targeted. The placenta is modelled as a single plane located at $300 \mathrm{~mm}$ from the body wall phantom and whose normal is pointing towards the insertion point. For this experiment, the simulated placenta can be placed on the left side, at $-15^{\circ}$, in the middle, at $0^{\circ}$, or on the right side, at $15^{\circ}$, from the user. Also, a desired number of anastomoses was set.

\section{B. Validation studies}

Three surgeons - all having an intermediate level of expertise in minimally invasive fetal surgery ${ }^{2}$ - were asked to use the above-mentioned experimental set up in order to perform a specific procedure detailed in Section IV-B.2.

1) Study objectives: With the validation studies, we aim to show a proof of concept using our proposed force sensor in a simulated clinical application. Second, we intend to show the force sensor's potential to investigate how the stress exerted on the body wall by the surgical instrument can be reduced in order to improve surgical outcome.

2) Surgical procedure: The clinical setting presented to the participants was that of TTTS [16]. The current treatment consists of selective laser coagulating the anastomoses followed by an equatorial dichorionization of the placenta [17]. For the experiment, the surgeon is asked to perform this treatment by following these steps (see Fig. 4b):

1. Insertion: insert instrument through cannula placed in the central hole of the body wall.

2. Targeting: move towards the left umbilical cord.

3. Pre-positioning: follow the blood vessels to reach the vascular equator.

4. Coagulation: selectively coagulate a predetermined number of anastomoses which are indicated by hollow circles in the VR system.

5. Solomonization: equatorial dichorionization by lasering the placenta in-between each ablated anastomose.

6. Retraction: removal of the instrument from the cannula. This is the end of the procedure.

3) Selected protocol: The three surgeons were each asked to execute 6 different procedures. The surgeons were asked to execute the above procedure with the set up in two different configurations : one with a rigid tip configuration without any robotic assistance, and another with a flexible tip configuration automatically bent by the robotic assistance which also provides damping and gravity compensation. For both configurations, referred to as rigid and flexible, the surgeons operated virtually three different placentas : a right side placenta with 6 anastomoses, a central placenta with 4 anastomoses, and a left side placenta with 6 anastomoses. Note that the chosen number of anastomoses was in the lower

\footnotetext{
${ }^{2} \mathrm{An}$ intermediate level is meant here a surgeon with surgical background but with no performance of a clinic fetal MIS as leading surgeon.
}

range of what is normally encountered in clinical reality [18], but allowed us to gather relevant data in a timely manner.

\section{Validation metrics}

In order to analyze the $3 \times 6$ experiments, measured forces and moments were re-formulated in components parallel to the body wall plane (planar components) and a component perpendicular (vertical component) to that plane. It was decided to only account for data that was generated after insertion of the instrument in the cannula. For comparison between the experiments, we selected the following metrics: maximum (Max), standard deviation (Std), and root mean square (RMS).

\section{Results \& Discussion}

Table IV displays the Max, Std, and RMS results for the planar forces obtained for each experimental configuration. All the results are expressed in Newtons. A first observation that can be made accross surgeons and for the rigid tip configuration is that, while the placenta pose is increasingly complex (e.g. left and right), the maximal and average planar forces also increase. Indeed, the average increase of forces goes up to $28 \%$ when operating a conventional rigid instrument on the left placenta pose. Hence, the force information seems to be able to indicate the level of complexity of the surgical task. This trend seems stronger than the 'learning' effect, which would forecast lower forces as one gets acquainted with the set up. Also, when comparing surgeon 3 with the others, one can notice a significant different handling style. Planar forces were substantially larger accross all metrics considered for the third surgeon. During the experiments, surgeon 3 was visibly manipulating the instrument more actively. This finding seems to confirm our expectation that handling techniques do translate in substantial variations in planar forces (and associated stresses at the fetal membranes).

Fig.5 shows the evolution of the planar force profiles over time when surgeon 2 executes the solomonization (continuous lasering) between two successive anastomoses on a left placenta. In green, the surgeon employed the rigid tip and in brown the flexible one. The differences in planar

TABLE IV: The planar forces for surgeons 1,2 and 3 in each configuration (all metric forces in Newton)

\begin{tabular}{|c|c|c|c|c|c|c|c|}
\hline & & \multicolumn{3}{|c|}{ Rigid tip } & \multicolumn{3}{|c|}{ Flexible tip } \\
\hline & & Central & Left & Right & Central & Left & Right \\
\hline \multirow{3}{*}{1} & $\operatorname{Max}$ & 8.91 & 9.76 & 10.11 & 5.49 & 7.10 & 8.98 \\
\hline & RMS & 3.04 & 4.47 & 2.39 & 2.74 & 2.93 & 2.68 \\
\hline & Std & 1.35 & 1.11 & 1.17 & 1.03 & 1.32 & 1.38 \\
\hline \multirow{3}{*}{2} & Max & 7.76 & 8.75 & 8.70 & 6.78 & 7.72 & 8.26 \\
\hline & RMS & 3.64 & 4.23 & 2.97 & 2.24 & 3.27 & 3.76 \\
\hline & Std & 1.23 & 1.73 & 1.19 & 1.14 & 1.44 & 1.62 \\
\hline \multirow{3}{*}{3} & $\operatorname{Max}$ & 9.77 & 10.75 & 11.33 & 10.29 & 12.91 & 12.09 \\
\hline & RMS & 4.06 & 5.08 & 4.98 & 4.48 & 7.63 & 3.61 \\
\hline & Std & 1.53 & 2.06 & 2.70 & 1.91 & 2.82 & 1.80 \\
\hline
\end{tabular}




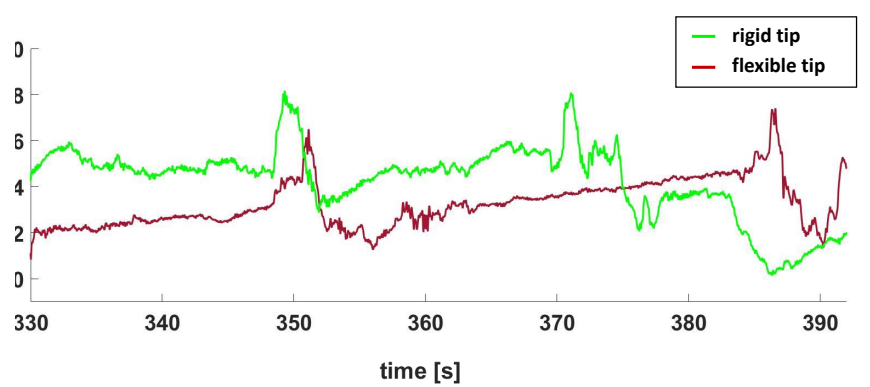

Fig. 5: Planar force profiles for surgeon 2 executing the solomonization between 2 consecutive anastomoses on a left placenta configuration. In green, using a rigid tip and in red using a flexible tip.

forces between the two tip configurations are clearly visible even for the naked eye. In Table IV, it can be observed that both the mean and maximum forces applied on the body wall have been significantly reduced when using the flexible configuration. Without haptics, the surgeon exerts about 1.5 more force in average. Only when repositioning the tool (this corresponds to peaks in each curve in Fig.5), the added-value from the novel technology is not apparent. These experiments seem to confirm that automatic haptic assistance and use of flexible instruments could play a role in reducing the stress applied on specific anatomical structures. Because of the low number of subjects, we cannot attribute this improvement to a specific factor (bending tip or haptics) at this point. However, these results show that this force sensor could help confirm this statement in a such dedicated study.

\section{CONCLUSION}

A new force sensor capable of measuring the interaction forces between the surgical instrument and the body wall in 6 DOFs during a simulated MIS interventions on an ex-vivo set up has been designed and interfaced. The design of the sensor allows attaching various synthetic, but also eventually biological tissue replicas. The sensor does not interfere with the normal instrument handling. In fact, surgeons participating in the experiments indicated to have received a very realistic haptic feel.

The performance of the new sensor has been characterised in detail. Whereas the accuracy of the sensor was found to be acceptable, a significant difference in quality of force measurement as function of the direction was observed. It is believed that the difference in quality is mere an artifact caused by the manufacturing/assembly of the sensor and/or the mounting on the test rig. This problem could be partially sorted by using a 6 DOFs rather than a 1 DOF load-cell during the characterization.

A study of simulated fetal surgery (TTTS) was performed by three surgeons to validate and emphasize the potential of the proposed device. The surgeons made use of a traditional rigid endoscope and of a flexible endoscope. For the latter, additional haptic support was provided, mainly in the form of added damping and gravity compensation. From the experi- ments, it could be observed that the combination of assisted technology did cause an important decrease of forces applied in-plain of the body wall compared to free-hand manipulation of the rigid instrument. At this point it is not possible to make any statement as to which of flexible endoscope, gravity compensation or added damping was contributing more to this promising result. A more detailed and expansive study would be needed to clarify this. Also, the here performed experiments made use of synthetic tissue. Therefore in a further stage it would be interesting to repeat the experiments making use of biological tissue as this would add to the realism of the experiment.

Large differences were observed between surgeons with similar level of expertise. Further elaborated studies with the proposed sensor seem useful to confirm this difference. Such experiments could also be used to acquire a better understanding of the difference in surgical technique. We are also looking forward to include expert fetal surgeons inside the user group to understand whether variability in results is normal or whether we can recognize experts from specific force/pose signatures.

Lastly, some first simple haptic schemes offering damping and gravity compensation have been used here. Further expansion can be done towards more sophisticated haptic schemes. Additionally a more in-depth study investigating in depth the impact of a certain haptic scheme versus another would be of use. Currently, simple metrics relying on planar forces were employed. However, expanding the set of metrics could show even stronger correlations between the performance and expertise of a surgeon. Such knowledge could then be incorporated in dedicated training schemes that are tailored to improve surgeon's skill at specific aspects.

\section{ACKNOWLEDGMENT}

This work was supported through an Innovative Engineering for Health award by Wellcome Trust [WT101957] and Engineering and Physical Sciences Reserach Council (EPSRC) [NS/A000027/1].

\section{REFERENCES}

[1] B. Davies, "A review of robotics in surgery," Proceedings of the Institution of Mechanical Engineers, Part H: Journal of Engineering in Medicine, vol. 214, no. 1, pp. 129-140, 2000.

[2] A. Darzi and S. Mackay, "Recent advances in minimal access surgery," British Medical Journal, vol. 324, no. 7328, pp. 31-34, 2002.

[3] P. Breedveld and M. Wentink, "Eye-hand coordination in laparoscopy - an overview of experiments and supporting aids," Minimally Invasive Therapy \& Allied Technologies, vol. 10, no. 3, pp. 155-162, 2001.

[4] Z. Lin, M. Uemura, M. Zecca, S. Sessa, H. Ishii, M. Tomikawa, M. Hashizume, and Takanishi, "Objective skill evaluation for laparoscopic training based on motion analysis," IEEE Transactions on Biomedical Engineering, vol. 60, no. 4, pp. 977-985, 2013.

[5] R. A. Quintero, "Twin-twin transfusion syndrome," Clinics in perinatology, vol. 30, no. 3, pp. 591-600, 2003.

[6] L. Maggio, S. Carr, D. Watson-Smith, B. O'Brien, V. Lopes, C. Muratore, and F. Luks, "Iatrogenic preterm premature rupture of membranes after fetoscopic laser ablative surgery," Fetal Diagn Ther, vol. 38, no. 1, pp. 29-34, 2015.

[7] J. Peirs, J. Clijnen, D. Reynaerts, H. Van Brussel, P. Herijgers, B. Corteville, and S. Boone, "A micro optical force sensor for force feedback during minimally invasive robotic surgery," Sensors and Actuators A: Physical, vol. 115, pp. 447 - 455, 2004. 
[8] P. Puangmali, H. Liu, K. Althoefer, and L. D. Seneviratne, "Optical fiber sensor for soft tissue investigation during minimally invasive surgery," in IEEE International Conference on Robotics and Automation, 2008, pp. 2934-2939.

[9] U. Seibold, B. Kubler, and G. Hirzinger, "Prototype of instrument for minimally invasive surgery with 6-axis force sensing capability," in Proceedings of the 2005 IEEE International Conference on Robotics and Automation, 2005, pp. 496-501.

[10] L. Howell, Compliant mechanisms. Wiley, 2001.

[11] S. Shimachi, Y. Hakozaki, T. Tada, and Y. Fujiwara, "Measurement of force acting on surgical instrument for force-feedback to master robot console," International Congress Series, 2003.

[12] N. Zemiti, G. Morel, T. Ortmaier, and N. Bonnet, "Mechatronic design of a new robot for force control in minimally invasive surgery," IEEE/ASME Transactions on Mechatronics, vol. 12, no. 2, pp. 143153, 2007.

[13] B. Willaert, D. Reynaerts, H. Van Brussel, and E. Vander Poorten, "Extracorporeal force measurement system for robot-assisted mis with 3d force feedback," in ICRA workshop: Advanced Sensing and Sensor Integration in Medical Robotics, 2009.

[14] J. Rosen, B. Hannaford, C. G. Richards, and M. N. Sinanan, "Markov modeling of minimally invasive surgery based on tool/tissue interac- tion and force/torque signatures for evaluating surgical skills," IEEE Trans. Biomed. Eng., vol. 48, no. 5, pp. 579-591, 2001.

[15] C. Gruijthuijsen, B. Rosa, H. Snyers, A. Engels, T. Vercauteren, J. Deprest, S. Ourselin, D. Reynaerts, and E. Vander Poorten, "Prototyping novel instruments for fetal surgery through virtual reality simulation and 3d printing," in Proceedings of the 5th Joint Workshop on New Technologies for Computer/Robot Assisted Surgery, 2015.

[16] L. Lewi, D. Van Schoubroeck, E. Gratac?s, I. Witters, D. Timmerman and J. Deprest, "Monochorionic diamniotic twins: complications and management options," Curr Opin Obstet Gynecol., vol. 15, no. 2, pp. 177-194, 2003.

[17] A. A. Baschat, J. Barber, N. Pedersen, O. M. Turan, and C. R. Harman, "Outcome after fetoscopic selective laser ablation of placental anastomoses vs equatorial laser dichorionization for the treatment of twin-to-twin transfusion syndrome," American journal of obstetrics and gynecology, vol. 209, no. 3, pp. 234-e1, 2013.

[18] D. Zhao, S. De Villiers, F. Slaghekke, F. Walther, J. Middeldorp, D. Oepkes, and E. Lopriore, "Prevalence, size, number and localization of vascular anastomoses in monochorionic placentas," Placenta, vol. 34, no. 7, pp. 589-593, 2013 